PREPARED FOR THE U.S. DEPARTMENT OF ENERGY, UNDER CONTRACT DE-AC02-76CH03073

PPPL-3903

PPPL-3903

UC-70

Stability of Microturbulent Drift Modes during Internal Transport Barrier Formation in the Alcator C-Mod Radio Frequency Heated H-mode

by

M.H. Redi, C.L. Fiore, W. Dorland, D.R. Mikkelsen, G. Rewoldt, P.T. Bonoli, D.R. Ernst, J.E. Rice, and S.J. Wukitch

November 2003
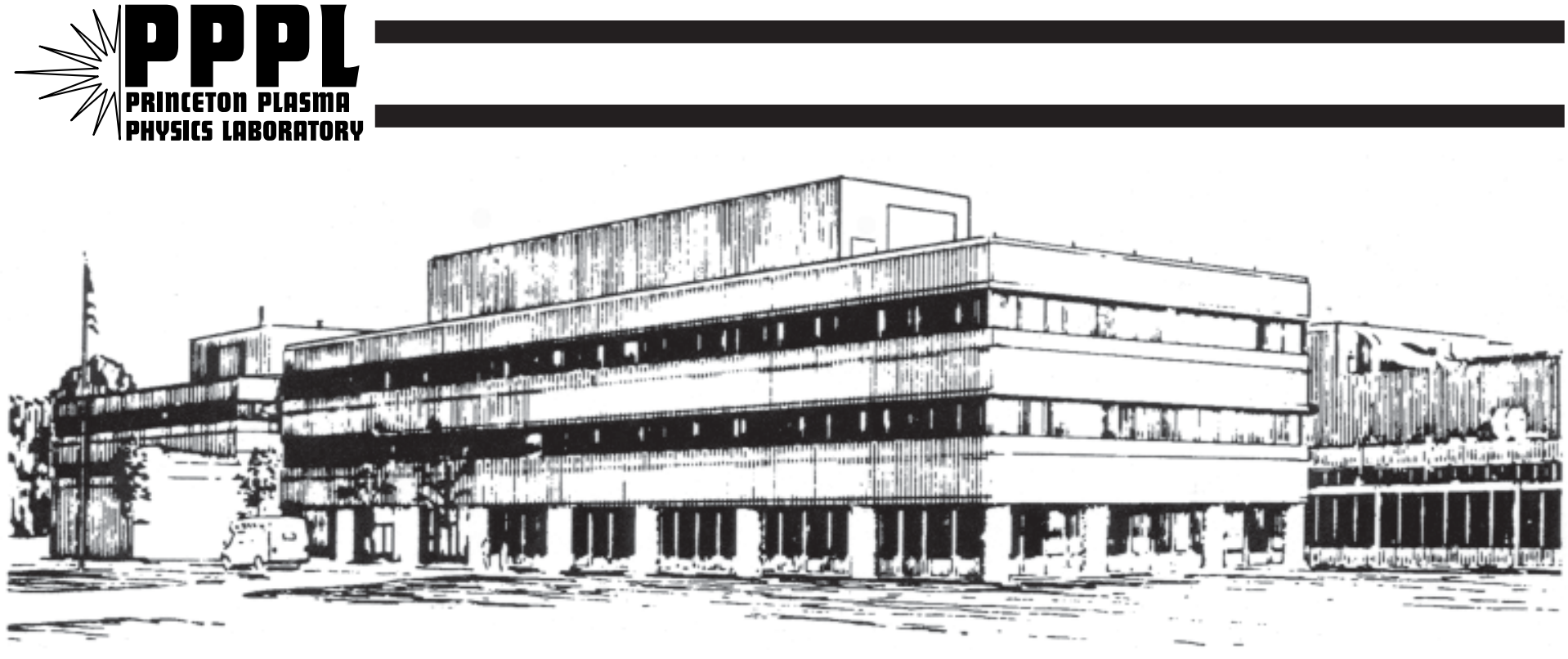

PRINCETON PLASMA PHYSICS LABORATORY PRINCETON UNIVERSITY, PRINCETON, NEW JERSEY 


\section{PPPL Reports Disclaimer}

This report was prepared as an account of work sponsored by an agency of the United States Government. Neither the United States Government nor any agency thereof, nor any of their employees, makes any warranty, express or implied, or assumes any legal liability or responsibility for the accuracy, completeness, or usefulness of any information, apparatus, product, or process disclosed, or represents that its use would not infringe privately owned rights. Reference herein to any specific commercial product, process, or service by trade name, trademark, manufacturer, or otherwise, does not necessarily constitute or imply its endorsement, recommendation, or favoring by the United States Government or any agency thereof. The views and opinions of authors expressed herein do not necessarily state or reflect those of the United States Government or any agency thereof.

\section{Availability}

This report is posted on the U.S. Department of Energy's Princeton Plasma Physics Laboratory Publications and Reports web site in Fiscal Year 2004. The home page for PPPL Reports and Publications is: http://www.pppl.gov/pub_report/

DOE and DOE Contractors can obtain copies of this report from:

U.S. Department of Energy

Office of Scientific and Technical Information

DOE Technical Information Services (DTIS)

P.O. Box 62

Oak Ridge, TN 37831

Telephone: (865) 576-8401

Fax: (865) 576-5728

Email: reports@adonis.osti.gov

This report is available to the general public from:

National Technical Information Service

U.S. Department of Commerce

5285 Port Royal Road

Springfield, VA 22161

Telephone: $1-800-553-6847$ or

(703) $605-6000$

Fax: (703) 321-8547

Internet: http://www.ntis.gov/ordering.htm 


\title{
Stability of microturbulent drift modes during internal transport barrier formation in the Alcator C-Mod radio frequency heated H-mode
}

\author{
M. H. Redi \\ Princeton Plasma Physics Laboratory, Princeton, NJ 08540, USA \\ C. L. Fiore \\ Plasma Science and Fusion Center, MIT, Cambridge, MA 02139, USA \\ W. Dorland \\ Institute for Plasma Research, U. MD, College Park, MD 20742, USA \\ D. R. Mikkelsen, G. Rewoldt \\ Princeton Plasma Physics Laboratory, Princeton, NJ 08540, USA \\ P. T. Bonoli, D. R. Ernst, J. E. Rice, S. J. Wukitch \\ Plasma Science and Fusion Center, MIT, Cambridge, MA 02139, USA
}

(Received

Recent H-mode experiments on Alcator C-Mod [I. H. Hutchinson, et al., Phys. Plas. 1, 1511 (1994)] which exhibit an internal transport barrier (ITB), have been examined with flux tube geometry gyrokinetic simulations, using the massively parallel code GS2 [M. Kotschenreuther, G. Rewoldt, and W. M. Tang, Comput. Phys. Commun. 88, 128 (1995)]. The simulations support the picture of ion/electron temperature gradient (ITG/ETG) microturbulence driving high $\square_{i} / \square_{e}$ and that suppressed ITG causes reduced particle transport and improved $\square_{i}$ on C-Mod). Nonlinear calculations for C-Mod confirm initial linear simulations, which predicted ITG stability in the barrier region just before ITB formation, without invoking $\boldsymbol{E} \square \boldsymbol{B}$ shear 
suppression of turbulence. Nonlinear fluxes are compared to experiment, which both show low heat transport in the ITB and higher transport within and outside of the barrier region.

(PACS numbers: 52.30.Gz, 52.35.Ra, 52.55.Fa) 


\section{INTRODUCTION}

Recent years have seen exciting and dramatic progress in the development and application of computational physics methods for predicting drift wave microturbulence and transport in magnetically confined plasma experiments as is evident in the reviews by Tang ${ }^{1}$, Horton $^{2}$ and Burrell ${ }^{3}$. Validation of gyrokinetic-based models of drift wave turbulence is being sought via comparative simulations of high performance plasma experiments for new understanding of transport. Such massively parallel, nonlinear, fully electromagnetic, nonadiabatic, gyrokinetic calculations are in the initial stages of application toward experimentally validated, global, nonlocal, first principles calculations of plasma transport.

When internal barriers to plasma particle and energy transport develop, high energy plasma is well confined, a necessary step toward economical fusion reactors. In this paper plasma conditions just before an internal transport barrier (ITB) is established on Alcator C$\mathrm{Mod}^{4}$ are analysed using the $\mathrm{GS2}^{5,6}$ gyrokinetic code. Because C-Mod is a toroidal magnetic confinement device with high toroidal field, high plasma density, and radio frequency (RF) heating, its transport characteristics are of special interest, being relevant to fusion reactor scenarios ${ }^{7}$. The linear ${ }^{8}$ and nonlinear ${ }^{9}$ simulations predict, just before ITB formation (at the "trigger time"), that plasma drift wave turbulence is suppressed in the plasma core, is quiescent where the ITB will form, and of classic ion temperature gradient character outside this region. Unlike ITBs on other magnetically confined fusion devices, this ITB is found to occur without either weak or reversed magnetic shear, and without strong plasma velocity shear ${ }^{10-12}$, the source of the usual $\boldsymbol{E} \boldsymbol{x} \boldsymbol{B}$ shear suppression of linear drift wave turbulence ${ }^{13-15}$.

We describe the experiment and physics analysis in Sec. II. In Sec. III are the microstability analysis results along with discussion of the gyrokinetic simulation approach, and 
comparison of simulated fluxes to those from transport analysis. A summary and conclusions are given in Sec. IV.

\section{THE EXPERIMENT: INTERNAL TRANSPORT BARRIER IN ALCATOR C-MOD RF H-MODE}

Following off-axis RF heating and establishment of an H-mode, C-Mod develops a reproducible ITB with a very steep electron density profile (see Figs. 1-2). Ion and electron thermal confinement are found from experimental analysis to be better than neoclassically predicted in the plasma core. A typical such experiment is analysed here, pulse \#1001220016. It is characterized by $R_{o}=0.69 \mathrm{~m}, B_{o}=4.5 \mathrm{~T}, I p=0.78 \mathrm{MA}$, and central electron density $\mathrm{n}_{\mathrm{e}}(0)=$ $4 \times 10^{20} / \mathrm{m}^{3}$. Deuterium majority ion and impurities of oxygen, carbon, boron and molybdenum give $<Z_{\text {eff }}>\sim 1.64$. Plasma central beta is less than $1 \%$. Toroidal rotation is found to reverse sign as the barrier is established.

The ITB exhibits steep, spontaneous density peaking, a reduction in particle transport occurring without a central particle source. The ITB development occurs in the early phase of a dual frequency RF experiment, which shows density control with central RF heating later in the discharge. Figure 2 is a plot of time slices of the radial profiles of the plasma electron density. We are interested in examining the "trigger time", $0.9 \mathrm{sec}$, just before the ITB is established, to identify the plasma conditions conducive to ITB formation. For this ICRF EDA H-Mode, the minority resonance is at $r / a \sim 0.5$ on the high field side, beginning at 0.7 secs. EDA refers to edge $\mathrm{D}_{\square}$ radiation, which is high for high performance H-modes at high densities and temperatures. Since the central temperature is maintained while the central density is increasing, this also suggests that a thermal transport barrier exists ${ }^{10}$, in addition to the barrier to particle transport 
evident in Fig. 2. Sawtooth heat pulse propagation experiments ${ }^{11}$ show that the ITB is limited to a very localized region (Fig 3.).

The experiment has been analysed with the TRANSP code $^{16}$, including a sawtooth model which causes the safety factor, $q$, to drop below unity by $\sim 10 \%$ at each sawtooth. The TRANSP results were processed with GS2_PREP ${ }^{17}$ for GS2 input, averaging over $50 \mathrm{~ms}$ at the time of interest. As the sawtooth period is $10 \mathrm{~ms}$, a sawtooth-averaged condition is used for the simulations. The key input variables for each radius simulated are found in Table I. The plasma had a normal shear profile, with q monotonic. Error in the experimental measurements of all data is estimated to be on the order of $10-20 \%$.

The impurity and minority ions at the "trigger" time are estimated to be $3 \%$ boron and $4 \%$ hydrogen. The walls of C-Mod are molybdenum, regularly coated with boron (from $\mathrm{D}_{2} \mathrm{~B}_{6}$ boronization) for edge density control. Examination of bolometer profiles indicates molybdenum levels $<0.1 \%$. The low $Z$ impurity level is estimated at 3\% from visible bremsstrahlung measurements, yielding $Z_{\text {eff. }}$ The impurity ion is identified as boron, although the low $Z$ impurities may be actually $2 \%$ boron and $1 \%$ carbon. Boron and carbon are so similar in collisional properties that only boron impurity ions are included in the simulations. No oxygen is thought to have been present. The hydrogen to deuterium ratio, $4 \%$ at the trigger time, was obtained from spectroscopic measurements of $\mathrm{D}_{\square}$ and $\mathrm{H}_{\square}$ radiation.

Radio frequency heating of the hydrogen minority causes the hydrogen temperature $\left(T_{h}=2 E_{h} / 3 k\right)$ to be peaked around the half radius ${ }^{18}$, with a less radially peaked hydrogen density profile. The ion distribution function is not thought to have a high energy RF tail, due to the high density and collisionality. $\boldsymbol{E} \square \boldsymbol{B}$ shearing rates can be estimated from measurements of toroidal 
rotation but at the time of interest, the toroidal rotation is near zero, changing from co to counter rotation as the ITB is established.

Electron temperature data for the TRANSP analysis was taken from Thomson scattering as the electron cyclotron emission (ECE) signal is cut off during the ITB because of rising high electron density. The density profile data was obtained from inverted visible bremstrahlung measurements, adjusted for the $Z_{\text {eff }}$ and temperature dependence. The plasma ion temperature profile in TRANSP was calculated on the basis of neutron data and the assumption that $\square$ is proportional to $\square_{i}^{\text {Chang-Hinton }}$. This leads to $T_{i}(r)$ being broader, and slightly lower than $T_{e}(r)$. While the high density of C-Mod suggests that $T_{i}=T_{e}$ would be a good assumption in the plasma core, the data are consistent with either ion thermal loss model.

\section{GYROKINETIC SIMULATIONS}

\section{A. GS2 microstability code}

The transport of particles and energy in high temperature fusion plasmas is widely believed to result from the turbulence of low frequency drift wave fluctuations. A complete, theory-based calculation of such microturbulence throughout the experimental plasma volume is not presently feasible. In this paper, results from drift wave microturbulence stability simulations along single flux tubes are reported, to test the concept for a specific experiment. The calculations were carried out with the GS2 gyrokinetic microinstability code ${ }^{5,6}$, which is

based on the electromagnetic nonlinear gyrokinetic equation ${ }^{19-23}$. This equation describes the evolution of fluctuations which satisfy

$$
\frac{\tilde{h}}{F_{0}} \sim \frac{e \tilde{\square}}{T} \sim \frac{\tilde{A}_{\| /}}{B \square} \sim \frac{\tilde{B}_{\|}}{B} \sim \frac{\square}{\square} \sim \frac{\square}{L}=\square<<1, \quad k_{/ /} L \sim k_{\square} \square \sim 1 .
$$


Here $\tilde{h}$ is the nonadiabatic part of the perturbed distribution function, $F_{0}$ is the equilibrium distribution function, $\tilde{\square}$ and $\tilde{A}_{/ /}$are the perturbed parts of the electrostatic and parallel vector potential, $\tilde{B}_{/ /}$is the perturbed parallel magnetic field, $B$ is the equilibrium magnetic field, $L$ is an equilibrium scale length (of density, temperature, or magnetic field), and $\square=e B /(m c)$ and $\square=v_{t} / \square$ are the cyclotron frequency and thermal gyroradius of a given particle species with thermal velocity $v_{t}^{2}=T / m$ and charge $e$. The simulations are performed in field-line-following coordinates using toroidal flux tubes ${ }^{24-26}$. In such coordinates, the nonlinear gyrokinetic equation may be written as

$$
\left(\frac{d}{d t}+\mathrm{v}_{/ /} \mathbf{b} \cdot \square+i \square_{d}\right) \tilde{h}=i \square_{\square}^{T} \square \square e \frac{\partial F_{0}}{\partial \square} \frac{\partial \square}{\partial t} .
$$

Here the distribution function $F_{0}=F_{0}(\square \square)$ depends only on the energy $\square=m v^{2} / 2$ and the flux surface label $\square$, where $\square$ is the equilibrium poloidal magnetic flux enclosed by the magnetic surface of interest. The total time derivative is given by $d_{t}=\partial_{t}+(c / B)[\square \cdot]$, where $[\because, \cdot]$ is the Poisson bracket. The perpendicular curvature and $\square B$ drifts are given by

$$
\square_{d}=\boldsymbol{k}_{\square} \cdot \boldsymbol{B} \square\left(m \mathrm{v}_{/ /}^{2} \boldsymbol{b} \cdot \square \boldsymbol{b}+\square \square B\right) /(B m \square),
$$

where $\square=\mathrm{mv}_{\square}{ }^{2} /(2 \mathrm{~B})$ and the fields are represented by

$$
\square=J_{0}\left(\square\left(\tilde{\square} \square \frac{\mathrm{v}_{/ /}}{c} \tilde{A}_{/ /}\right)+\frac{J_{1}(\square}{\square} \frac{m \mathrm{v}_{\square}^{2}}{e} \frac{\tilde{B}_{/ /}}{B} .\right.
$$

Here, $\square=k_{\square} v_{\square} / \square$ and $\square_{\square}^{T}=n_{0} c \partial_{\square} F_{0}$, where $n_{0}$ is the toroidal mode number of the perturbation. The self-consistent electromagnetic field fluctuations are computed from the gyrokinetic Poisson-Ampere equations,

$$
\begin{aligned}
& \square_{\square}^{2} \tilde{\square}=4 \square \underset{s}{\square} e \square d^{3} r\left[e \tilde{\square} \frac{\partial F_{0}}{\partial \square}+J_{0}(\square \tilde{h}],\right. \\
& \square_{\square}^{2} \tilde{A}_{/ /}=\square \frac{4 \square_{c}}{\square} \square d^{3} r\left[e v_{/ /} J_{0}(\square \tilde{h}],\right.
\end{aligned}
$$




$$
\frac{\tilde{B}_{\|}}{B}=\square \frac{4 \square}{B^{2}} \square\left[d_{s}^{3} r\left[m v_{\square}^{2} \frac{J_{1}(\square}{\square} \tilde{h}\right]\right.
$$

The Bessel functions $J_{0}$ and $J_{1}$ arise because equations 5-7 are formulated in particle space $\mathbf{x}$, rather than in gyrocenter space $\mathbf{R}$. We retain the Debye-shielding term $\square^{2} \square \tilde{\square}$ in Poisson's equation, since the electron Debye length $\square_{D e}$ can be comparable to $\square_{\mathrm{e}}$ in laboratory fusion experiments. Of course this term can be neglected when only ion-scale instabilities are studied.

GS2 is a nonlinear generalization of a widely used gyrokinetic stability code ${ }^{5}$. An operator splitting scheme is use, so that the linear terms, including equations 5-7, may be treated implicitly ${ }^{5}$. The nonlinear terms are evaluated with a dealiased pseudospectral algorithm in the plane perpendicular to the field line. A second-order Adams-Bashforth scheme is used to advance the nonlinear terms in time. Non-uniform coordinate meshes are used in velocity space to improve the resolution, particularly for the trapped-passing boundary. A small amount of upwind diffusion is typically used, only in the direction along the field line. In the absence of upwind diffusion, the algorithm is second-order accurate in space and time. Good parallel performance is achieved by employing multiple-domain decomposition in four of the five dimensions at all times.

This initial value code solves for the perturbed eigenfunctions of the electrostatic potential $\square$ and the components of the electromagnetic potential, $A$, parallel and perpendicular to the field line direction. The ballooning representation along a field line is chosen for model simplicity with, for example, the perturbed electrostatic potential eigenfunction written

$$
\tilde{\square}(r, \square, \square, t)=\exp [i n \square \square i n q(r) \square] \square_{p=\square}^{p=} \tilde{L}(\square \square 2 \square p, r, t) \exp [i n q(r) 2 \square p]
$$


Here $(r, \square, \square)$ are the usual radial, poloidal angle and toroidal angle coordinates. To illustrate the highly nonlinear nature of the drift wave microstability calculation, the linearized gyrokinetic equation, using the "s- $\square$ " MHD equilibrium is written ${ }^{5}$, neglecting component $A_{\square}$,

$$
\frac{\partial}{\partial t} \tilde{g}_{s}+\frac{v_{\mu}}{q R} \frac{\partial}{\partial \square} \tilde{g}_{s}+i \square_{d s} \tilde{s}_{s}+C\left(\tilde{g}_{s}\right)=\frac{e_{s}}{T_{s}} F_{m s}\left(\frac{\partial}{\partial t}+i \square_{*_{s}}^{T}\right)\left\{\left[\tilde{\square}(\square) \square \frac{v_{/ \prime}}{c} \tilde{A}_{/ /}(\square)\right] J_{0} \square i \frac{\mathcal{V}_{\square}}{c} \tilde{A}_{\square}(\square) J_{1}\right\}
$$

where

$$
\begin{aligned}
& \tilde{g}_{s} \equiv \tilde{f}_{s}+\left(\frac{e_{s}}{T_{s}}\right) F_{m s} \tilde{\square}(\square), \\
& \square_{d s}=\square_{*_{s}}\left(L_{n s} / R\right)\left(E / T_{s}\right)\left(1+v_{/ /}^{2} / v^{2}\right)\{\cos \square+[\tilde{s} \square \square \square \sin \square] \sin \square\} \\
& k_{\square}=\square n q / r \\
& k_{\square}=k_{\square}\left\{1+[\tilde{s} \square \square \square \sin \square]^{2}\right\}^{1 / 2} \\
& \tilde{s} \equiv(r / q)(d q / d r) \\
& \square \equiv \square q^{2} R(d \square / d r) \\
& \square_{*_{s}}^{T} \equiv \square_{*_{s}}\left\{\left[1+\square_{s}\left[E / T_{s}\right) \square 3 / 2\right]\right\} \\
& J_{0} \equiv J_{0}\left(k_{\square} v_{\square} / \square_{s}\right) \\
& \square_{*_{s}} \equiv k T_{s}\left(\square n_{s} / n_{s}\right) / e_{s} B \\
& L_{n s} \equiv\left(n_{s} / \square n_{s}\right) \\
& \square_{s} \equiv d \ell n T_{s} / d \ell n n_{s}
\end{aligned}
$$

In general, driving forces for the microturbulence arise from temperature and density gradients in $\square *$ s, mediated by the effects of passing ions and of trapped electrons. Stabilization of turbulence is achieved through high values of shear, $q, \square[$ impurities, collisional effects, and nonzero $T_{e}$. The competition among many driving and stabilizing forces mandates computational methods to analyse a particular experimental situation.

\section{B. SIMULATION PARAMETERS AND RESULTS}

We examine only the time just before the ITB is established, and the plasma density peaking begins. Three zones characteristic of the experimental conditions are simulated (Fig. 2): the plasma core at $\sim 0.25 r / a$, the region where the ITB occurs at $\sim 0.45 r / a$ and outside the ITB at 
$\sim 0.65 r / a$. The calculations were carried out at the USDOE NERSC Cray T3E and IBM RS/6000SP computers using a total of $\sim 50,000$ hours, with runs typically making use of 40-256 parallel processors.

\section{Linear simulations}

The linear simulations are fully electromagnetic, include the nonadiabatic electron response, and four plasma species: electrons, deuterium, boron impurity and fast hydrogen ions. They covered the full range of drift mode wavevectors, including ion temperature gradient mode (ITG), trapped electron mode (TEM) and electron temperature gradient mode (ETG) wavelengths, $k_{\square} \square_{i}=0.1$ to 80 (Figs. 4, 5). We will denote microturbulence from $k_{\square} \square_{i}=0.1$ to 1 by ITG-TEM, as these instabilities are hybrid modes characterized by both ITG and TEM behavior. From $k_{\square} \square_{i}=2$ to 10 the instabilities are TEM, while above $k_{\square} \square_{i}=10$ the microturbulence is pure ETG, driven by passing electrons only. The simulations solve the gyrokinetic Vlasov-Maxwell system and are run out for 10,000 time steps, until the microinstability growth rates, $\square$ and real frequencies, $\square$, are verified to have converged and the usual measure of the electrostatic potential, $\ln \mid \square^{2}$, is verified to be linearly increasing, in cases that are designated unstable.

The stability analysis shows that in the barrier region $(r / a \sim 0.45)$ no ITG-TEM mode is strongly growing for $0.2<k_{\square} \square_{i}<0.8$, while outside the ITB region a clear ITG-TEM signature is found. In the plasma core there are no strongly growing modes at $0.5 \square k_{\square} \square_{i} \square 0.8$ and only poorly resolved modes with $\square<0$, rotating in the electron diamagnetic direction, are unstable at $k_{\square} \square_{i} \square 0.4$. The apparently unstable mode at $k_{\square} \square_{i}=0.1$ is not converged and does not have a well defined eigenfunction. At higher values of $k_{\square} \square_{i}$, the TEM (usually found near $k_{\square} \square_{i} \sim 1$ ) is stable 
everywhere. The ETG (peaked at $k_{\square} \square_{i} \sim 25$ ) is strongly unstable outside and at the barrier, and is stable in the core.

Anomalous $\square_{i}$ is associated with unstable ITG-TEM, so that we expect reduced ion thermal confinement at, and within the ITB. TRANSP analysis shows that $\square_{\text {eff }}$ drops inside the $\mathrm{ITB}^{10}$. Anomalous $\square_{e}$ is associated with strong ETG instability, and the mixing length model would predict $\square_{e}$ at the ITB to be half that outside the barrier. Sawtooth heat pulse propagation measurements of similar experiments have shown that the effective $\square^{\text {peatpulse }}$ is reduced (by factor $\sim 10$ ) in a narrow radial region of $\sim 1 \mathrm{~cm}$, located near the foot of the particle barrier, but not necessarily within the barrier ${ }^{1}$. Reduced microinstability growth rates predicted at the barrier are consistent with the observed reduced transport.

The sensitivity of the microturbulent stability in the ITB region has been examined through the response of the calculated real frequencies and growth rates to variation of specific driving forces across the plasma. Figure 6 shows the radial variation of the dimensionless drift mode driving and stabilizing parameters for the experiment at the trigger time. We find that $\square_{i}$ increases as $r / a$ increases, as does the normalized electron temperature gradient. These and the increasing inverse gradient for the primary impurity, boron (3\%), are consistent with stabilized ITG-TEM in the core. Removal of the boron impurity, while maintaining charge neutrality with $Z_{\text {eff }}=1$ destabilizes the ITG-TEM in the barrier region. Similarly, removal of the $4 \%$ hydrogen species while maintaining ambipolarity also destabilizes the ITG-TEM mode in the barrier region.

It is found in this case, that either decreasing the plasma electron density gradient or increasing the plasma electron temperature gradient causes the ITG-TEM mode to be destabilized in the transport barrier region. Near marginal stability where gyrokinetic 
calculations, rather than fluid calculations are needed, the instability is expected to grow linearly according to $\square=A \square T / T+B n / \square n$, where $A$ and $B$ depend on specific plasma conditions. Far from marginal stability the fluid approximation is useful; the instability is strong, becoming pressure gradient driven so that temperature and density gradients add up with equal weight $\checkmark\left(1+\square_{i}\right)$. Near marginal stability, as in this case, the ITG-TEM is ion temperature gradient driven. The behavior of the ITG-TEM mode is complex and controlled by complex nonlinear equations, as seen in Sec. IIIA. Romanelli ${ }^{27}$ has shown, with kinetic theory, that density peaking may stabilize or destabilize the ITG-TEM mode, depending on plasma collisionality, trapped electron fractions and $\mathrm{T}_{\mathrm{i}} / \mathrm{T}_{\mathrm{e}}$.

For the C-Mod ITB trigger time, we find the growth rates in the barrier region are more strongly elevated (factor 25) by doubling $\square T / T$ than by reducing $\square n / n$ by two. The measured dimensionless, normalized temperature and density gradients are 2.0 and 0.6 (Table I). Critical temperature gradients have been examined (Fig. 7) and show that in the barrier region at the trigger time, the plasma is far above destabilization through reduced density gradients, $\left(\mathrm{a} \square n_{e} / n_{e}\right)_{c} \sim 0.35$, but is very close to marginal stability considering only increased temperature gradients, $\left(\mathrm{a} \square T_{e} / T_{e}\right)_{c} \sim 2$. Stability of the ITG-TEM mode is accomplished by being far above the critical density gradient, although the plasma appears to be still in H-mode (Fig. 2).

\section{Nonlinear Simulations}

Nonlinear electrostatic simulations were carried out for the three zones, under the same initial conditions. For this low beta experiment, there should be no difference in the computed results, but electrostatic calculations are more efficient, and required 1/3 the computational cost of the corresponding nonlinear electromagnetic calculations. 
Just as the linear calculations show stable long wavelength turbulence at the ITB region, without invoking suppression by $\boldsymbol{E} \square \boldsymbol{B}$ shear at the trigger time, the nonlinear simulations of the C-Mod plasma also show quiescent microturbulence in the ITG-TEM range of frequencies in the barrier region, just before ITB formation (Fig. 8). The simulations use four values of $k_{\square}$ and 23 values of $k_{r}$. In the plasma core, weak turbulence is predicted, with saturation occurring along with the development of a $77 \mathrm{kHz}$ Geodesic Acoustic Mode (GAM) (see Fig. 8). A high frequency core mode at $80 \mathrm{kHz}$ for a similar C-Mod experiment has been found with $\mathrm{ECE}^{28}$. The simulated mode is a stable mode of the plasma, excited as a damped computational mode. The GAM depends only on the device size and the plasma temperature. In the experiment such a stable mode may be driven unstable by RF heating. The only well resolved linearly unstable mode in this frequency range in the simulations is an ITG-TEM mode at about $50 \mathrm{kHz}$, outside the ITB region.

In Figs 9-11 are shown the results for the nonlinear evolution of the plasma quasineutrality, $\left(n_{e}-n_{d^{-}} 5 n_{b}-n_{h}\right) / n_{e}$, and for the particle and heat fluxes of the electron, deuterium and boron ion species. We find that the quasineutrality in the plasma core is maintained to less than $1 \%$, to much less than $1 \%$ in the barrier region, and to about $1 \%$ outside the barrier. In the plasma core, the particle flux is outward, with electron and deuterium fluxes of similar magnitude, and the heat flux is also outward, with the electron heat flux being greater than the deuterium heat flux. In the barrier region, before formation, the particle flux from electrons and deuterium is inward, with electron and deuterium fluxes of similar magnitude, while the heat flux is outward, with the deuterium heat flux being greater $(\sim 3 x)$ than the electron heat flux. Outside the ITB region, the particle flux is inward, with electron flux $\sim 30 \%$ greater than the deuterium flux; the heat flux is outward, with the deuterium heat flux being much larger $(\sim 5 \mathrm{x})$ than the 
electron heat flux. The boron carries negligible particle and heat flux. Bursting behavior is evident in the fluctuating microturbulence. Similar results have been found by Ernst, et al. ${ }^{29}$.

In Table II are the particle and heat fluxes from the gyrokinetic calculations and from the TRANSP experimental analysis. Although this is not a steady state experimental condition, the correlation time of the fluctuations will be of the order of microseconds, much shorter than the time scale of the changing plasma (msec), and it is worthwhile to compare the fluxes in Table II. The experimental fluxes are obtained from the TRANSP analysis at the time of interest, for radial zones of width $1 / 20^{\text {th }}$ of the minor radius. We find qualitative agreement of the simulated and experimental fluxes (within factors of 10), excepting for $S_{e}, S_{i}$ and $Q_{i}$ outside the ITB, which are overestimated by a factor $\sim 35$.

These can be compared to results from gyrofluid ${ }^{14}$ and recent gyrokinetic simulations ${ }^{15,29,30}$. For the DIII-D L-mode, gyrofluid calculations ${ }^{14}$ including shear flow corrections, underestimated particle fluxes by less than a factor of five, and agreed with heat flux data within a factor of two for both ions and electrons. In simulations ${ }^{29}$ of highly anomalous experiments on DIII-D with extreme reversed shear, and on JET including a current hole, gyrokinetic calculations, not corrected for shear flow, found that the simulated and measured plasma fluxes differed by factors as high as 40 to $10^{3}$. Nonlinear, global gyrokinetic simulations $^{30}$ of DIII-D L-mode discharges have matched the measured energy diffusivities within experimental uncertainty, about a factor of two for ions and about $20 \%$ for electrons. The approximate agreement of our simulated and measured fluxes within $r / a=0.5$ may be due to there being no need for shear flow in suppressing plasma turbulence in this C-Mod case, the rotational velocity being near zero. 
Table II also gives the values of the heat and particle diffusivities and the TRANSP values. The TRANSP analysis does not have the radial resolution found in individual experimental diagnostics, such as seen in Fig. 3. The electron heat diffusivities agree within an order of magnitude within and at the ITB region, supporting the drift wave model for this transport coefficient. Neoclassical contributions to ion diffusivities would account for some of the difference between experimental and calculated values of $\square_{i}$, although again outside the ITB the simulations overestimate the transport coefficients. It is important to recall that the GS2 input parameters have experimental error of $10-20 \%$, that small changes in plasma profiles can cause large differences in predicted turbulence, that for this case the low toroidal velocity measurement is at one point only and that this is not a steady state comparison. Good confinement is found in these simulations to result from high values of $\square n / n$ and low $\square T / T$ in the ITB region, which stabilize the turbulent microstabilities near the plasma core.

\section{Discussion}

The gyrokinetic model calculations show that just before ITB formation, conditions have already been established for which a peaked density profile will occur and be sustained. The Ware pinch provides sufficient fueling to account for a sustained ITB peaked density profile ${ }^{18}$. Since we find no strong drift wave instabilities at the ITB region, microturbulent driving forces are not strong enough to provide the usual anomalous transport across the barrier region. Outside the core plasma, ITG-TEM and ETG drift modes are linearly unstable.

Identification of the driving forces responsible for drift wave microstability in the barrier region before the ITB appears, was explored by examining the effects of increased gradients for the electron, ion, impurity density and temperatures as well as magnetic shear. It is found that increases in the normalized electron temperature gradient caused the largest destabilization of the 
ITG-TEM mode in the barrier region at the trigger time. Linear sensitivity studies show that increased normalized temperature gradients and reduced normalized density gradients as well as increased plasma safety factor at the ITB location, would destabilize the ITG-TEM mode frequencies and would likely prevent ITB formation.

This suggests that the ITB is triggered by reduction in the normalized electron temperature gradient driving force for the ITG-TEM and ETG microstability when off-axis RF heats the plasma locally. This would explain the observation of ITB with off-axis but not on-axis $\mathrm{RF}$, as due to weaker $\left(\square T_{e}\right) / T_{e}$ at the barrier region. The C-Mod ITB studied here formed spontaneously, during an off-axis RF-heated H-mode. This occurs during the EDA H-mode, characterized by edge $D_{\square}$ radiation, but not during the elm-free H-mode, which occurs at lower plasma density. Microstability analysis for the elm-free H-mode, which does not lead to an ITB with similar off-axis RF heating, as well as for the trigger time of the spontaneously occurring ohmic H-mode ITB $^{10}$ will be important to contrast to this case, to clarify the ITB formation process. Weaker $\left(\square T_{e}\right) / T_{e}$ at the barrier region does not occur at the trigger time in the ohmic $\mathrm{H}-$ mode. Because there are so many driving forces, which can nonlinearly affect drift wave microstability, it would not be surprising to find that different processes are responsible for ITB formation in different experimental scenarios.

We find that microturbulence is suppressed in the ITB region without recourse to velocity or magnetic shear turbulence suppression, consistent with experimental measurements and transport analysis. Nonlinear calculations also show greatly reduced microturbulence at the location for formation of the barrier, before indications of changes in temperature and density are apparent. Higher transport outside and within the ITB region is found from both simulations and experimental analysis. 


\section{CONCLUSION}

The ITB during off-axis RF heating on C-Mod has been examined with gyrokinetic calculations in flux tube geometry. Linear and nonlinear calculations, including electrostatic and fully electromagnetic treatments, with the complete electron response, and following four plasma species, show ITG-TEM microturbulence is already suppressed in the ITB region before formation, at the trigger time, without recourse to the usual requirements of velocity or magnetic shear. The microstability analysis is qualitatively consistent with the experimental transport analyses, showing high transport outside and inside the ITB region before formation. Strong ITG-TEM and ETG drift wave turbulence are identified outside the barrier region, with reduced ETG (short wavelength) turbulence found in the ITB region. Nonlinear calculations support the linear stability results and demonstrate the saturation of core, ITB and outer plasma microturbulence.

The microstability analysis shows that, in this case, linear, flux tube calculations are sufficient for understanding the microscopic physics of drift mode ITB turbulence suppression on C-Mod. Although simulation of steady state plasma fluxes and transport coefficients are required for meaningful code benchmarking and validation against experiment, these calculations of a transient plasma confirm a testable and fairly successful model, as found in Table II and comparing Figs. 3 and 8. Better diagnostics would be desirable since the drift wave instabilities are extremely sensitive to the plasma profiles and the magnetic shear. While the heuristic picture provided by linear calculations is supported by the nonlinear results, detailed calculations of particle and heat fluxes are only in agreement with measurements within about an order of magnitude. It is interesting that recent flux tube, fully electromagnetic gyrokinetic 
simulations of microturbulence on the National Spherical Tokamak Experiment (NSTX) ${ }^{31}$ do not reflect so clearly the connection between drift mode instability and observed transport ${ }^{32}$. The tokamak paradigm of transport due to drift mode microturbulence may need modification in application to the spherical torus and other fusion geometries.

The conditions for formation of this type of ITB on C-Mod, analysed with gyrokinetic microstability calculations, show that $\boldsymbol{E} \boldsymbol{x} \boldsymbol{B}$ shear is not required. Future work may identify experimentally verifiable trigger mechanisms. In this case, reduced $\left(\square T_{e}\right) / T_{e}$ appears to be the primary stabilizing force on the ITG-TEM mode, although many other plasma parameters have been shown to be destabilizing. In other types of ITB experiments, one or more other plasma conditions may be controlling barrier formation. Establishment of ITB without the requirement of $\boldsymbol{E} \boldsymbol{x} \boldsymbol{B}$ shear, as for this experiment, may yield practical and economic benefits for fusion reactor operation.

\section{ACKNOWLEDGEMENT}

We are glad to acknowledge the experimental and diagnostic teams at Alcator C-Mod, which provided the data and basis for the analysis in this paper. We especially wish to acknowledge discussions with S. Wolfe, B. Lipschultz, J. Terry and M. Greenwald at MIT. Valuable and interesting conversations with E. Belli, R. Budny, G. Hammett, T. S. Hahm and W. Lee of Princeton Plasma Physics Laboratory, Princeton University, and C. Bourdelle, Euratom-CEA, France, were key to completing this study. We also thank G. Schilling, PPPL, and E. Marmar and M. Porkolab, MIT, for their support. Research was carried out under U. S. DOE Contract DE-AC02-76CH03073. 


\section{References}

[1] W. M. Tang, Nucl. Fusion, 18 (1978) 1089.

[2] W. Horton, Rev. Mod. Phys. 71 (1999) 735-776.

[3] K. Burrell, Phys. Plas. 4 (1997) 1499.

[4] I. H. Hutchinson, R. L. Boivin, F. Bombarda et al., Phys. Plas. 1, 1511 (1994).

[5] M. Kotschenreuther, G. Rewoldt and W. M. Tang, Comp. Phys. Comm. 88, 128 (1995).

[6] W. Dorland, F. Jenko, M. Kotschenreuther and B. N. Rogers, Phys. Rev. Lett. 85, 5579 (2000).

[7] “ITER Physics Basis”, Nuclear Fusion, 39 (1999) 2137-2638.

[8] M. H. Redi, C. Fiore, P. Bonoli, et al., Proceedings EPS (2002), Montreux, Switzerland, Paper 1.082.

[9] M. H. Redi, R. Bell, P. Bonoli et al., Proceedings EPS (2003), St. Petersburg, Russia, Paper P4.94.

[10] C. L. Fiore, J. E. Rice, P. T. Bonoli, et al., Phys. Plas. 8, 2023 (2001).

[11] S. Wukitch, R. L. Boivin, P. T. Bonoli, et al., Phys. Plas. 9, 2149 (2002).

[12] J. E. Rice, P. T. Bonoli, E. Marmar, et al., Nuclear Fusion 42 (2002) 510.

[21] R. E. Waltz, G. D. Kerbel, J. Milovich, G. W. Hammett Phys. Plas. 22408 (1995).

[22] D. W. Ross, R. V. Bravenec, W. Dorland, et al. Phys. Plas. 9 (2002) 177-184.

[23] R. V. Budny, R. Andre, A. Becoulet, et al. Plasma Physics and Controlled Fusion 44 (2002) 1215-1228.

[24] R. J. Hawryluk, in Physics of Plasmas Close to Thermonuclear Conditions, edited by B. Coppi, G. G. Leotta, D. Pfirsch, R. Pozzoli, and E. Sindoni (Peramon, Oxford, 1980), Vol. 1, P. 19. 
[25] D. Ernst, R. E. Bell, M. G. Bell, et al. Phys. Plas. 7 (2000) 615-625.

[26] P. Bonoli, R. L. Boivin, M. Brambilla, et al, $18^{\text {th }}$ IAEA, Sorrento, Italy, IAEA-CN-77, EXP4/01 (Oct.,2000.)

[19] T. M. Antonsen and B. Lane, Phys. Fluids 23, 1205 (1980).

[20] E. A. Frieman and L. Chen, Phys. Fluids 25, 502 (1982).

[21] R. G. Littlejohn, J. Plasma Phys. 29, 111 (1983).

[22] T. S. Hahm, W. W. lee and A. Brizard, Phys. Fluids 31, 1940 (1988).

[23] A. Brizard, J. Plasma Phys. 41, 541 (1989).

[24] S. C. Cowley, R. M. Kulsrud and R. Sudan, Phys. Fluids B3, 2767 (1991).

[25] K. V. Roberts and J. B. Taylor, Phys. Fluids 8, 315 (1965).

[26] M. A. Beer, S. C. Cowley and G. W. Hammett, Phys. Plasmas 2, 2687 (1995).

[27] F. Romanelli and S. Briguglio, Phys. Fluids B2 (1990) 754.

[28] A. Lynn, P. E. Phillips, A. E. Hubbard, S. J. Wukitch, IAEA H-mode Workshop, San Diego, CA (to be published 2003).

[29] D. R. Ernst, P. T. Bonoli, C. L. Fiore, et al., $44^{\text {th }}$ Annual Meeting of the Division of Plasma Physics (2002) Orlando FL, Bull. Am. Phys. Soc. V.47 No 9 p. 139, GO1.003:"Mechansims for Internal Transport Barrier Control in Alcator C-Mod”. D. R. Ernst, submitted to Phys. Plas. (2003).

[30] J. Candy and R. E. Waltz, Phys. Rev. Lett. 91 (2003) 045001-1.

[31] M. Ono, S. M. Kaye, Y.-K. M. Peng et al. Nucl. Fusion, 40, 557 (2000).

[32] M. H. Redi, W. Dorland, J. Candy, et al., $45^{\text {th }}$ Annual Meeting of the Division of Plasma Physics (2003) Albuquerque, NM, Bull. Am. Phys. Soc. KO1.007, "Gyrokinetic Calculations of Microturbulence and Transport in C-Mod and NSTX". "Gyrokinetic Calculations of 
Microturbulence and Transport in NSTX H-mode", M. H. Redi, S. M. Kaye, W. Dorland, et al., in preparation. 
Table I. Plasma parameters for microturbulence simulation of three zones at the trigger time for ITB formation of off-axis RF heated H-mode on C-Mod

\begin{tabular}{|c|c|c|c|}
\hline Parameter & $r / a \sim 0.25$ & 0.45 & 0.65 \\
\hline$q$ & 0.998 & 1.3 & 2.00 \\
\hline$\hat{s}$ & 0.12 & 0.87 & 1.52 \\
\hline$n_{d} / n_{e}$ & 0.80 & 0.80 & 0.80 \\
\hline$n_{b} / n_{e}$ & 0.03 & 0.03 & 0.03 \\
\hline$n_{h} / n_{e}$ & 0.04 & 0.04 & 0.04 \\
\hline$T_{e} / T_{d}$ & 1.04 & 0.87 & 0.95 \\
\hline$T_{d} / T_{d}$ & 1.00 & 0.99 & 1.01 \\
\hline$T_{b} / T_{d}$ & 1.00 & 0.99 & 1.01 \\
\hline$T_{h} / T_{d}$ & 1.32 & 4.57 & 1.57 \\
\hline$a_{r e f} \square \square n_{e} / n_{e}$ & 0.57 & 0.60 & 0.06 \\
\hline$a_{r e f} \square \square n_{d} / n_{d}$ & 0.57 & 0.60 & 0.06 \\
\hline$a_{r e f} \square \square n_{b} / n_{b}$ & 0.57 & 0.60 & 0.06 \\
\hline$a_{r e f} \neg \square n_{h} / n_{h}$ & 0.58 & 0.60 & 0.05 \\
\hline$a_{r e f} \square T_{e} / T_{e}$ & 1.18 & 2.00 & 2.76 \\
\hline$a_{r e f} \square T_{d} / T_{d}$ & 0.49 & 1.75 & 3.24 \\
\hline$a_{r e f} \square \square T_{b} / T_{b}$ & 0.50 & 1.75 & 3.25 \\
\hline$a_{r e f} f T_{h} / T_{e}$ & 0 & 0 & 0 \\
\hline$\square$ & 0.28 & 0.46 & 1.21 \\
\hline
\end{tabular}




\begin{tabular}{|c|c|c|c|}
\hline$\square_{d}$ & 0.24 & 0.24 & 0.24 \\
\hline$\square b$ & 0.25 & 0.25 & 0.25 \\
\hline$\square h$ & 0 & 0 & 0 \\
\hline$T_{r e f}(\mathrm{keV})$ & 1.16 & 0.97 & 0.54 \\
\hline$a_{r e f}(\mathrm{~m})$ & 0.22 & 0.22 & 0.22 \\
\hline$n_{r e f}=n_{e}\left(\mathrm{~m}^{-3}\right)$ & $3.2 \times 10^{20}$ & $2.8 \times 10^{20}$ & $2.7 \mathrm{ex} 10^{20}$ \\
\hline$\square_{r e f}$ & 0.007 & 0.005 & 0.003 \\
\hline Freq norm $=\left(T_{r e f} / m_{r e f}\right)^{0.5} / a_{r e f}\left(\mathrm{sec}^{-1}\right)$ & $1.1 \times 10^{6}$ & $9.8 \times 10^{5}$ & $7.3 \times 10^{5}$ \\
\hline$\square_{r e f}$ norm $=k_{\square}^{-1}(\mathrm{~cm})$ & 0.120 & 0.119 & 0.094 \\
\hline
\end{tabular}


Table II. Heat and particle fluxes across three flux surfaces of the pre-ITB plasma from GS2 gyrokinetic calculations and TRANSP physics analysis. Ion fluxes are combined results for deuterium and boron. The zones are at $r / a \sim 0.25,0.45$ and 0.65 . $\square_{i}^{\text {neoclassical }}$ is $\sim 0.3 \mathrm{~m}^{2} / \mathrm{sec}$.

\begin{tabular}{|c|c|c|c|c|c|c|c|}
\hline \multirow[b]{2}{*}{$\overline{G S 2}$} & \multicolumn{2}{|c|}{$\begin{array}{cc}S_{i} & S_{e} \\
\left(10^{20} / \mathrm{sec}\right)\end{array}$} & \multicolumn{2}{|c|}{$\begin{array}{c}Q_{i} \quad Q_{e} \\
(\mathrm{MW})\end{array}$} & \multicolumn{2}{|c|}{$\begin{array}{cc}\square_{i} \\
\left(\mathrm{~m}^{2} / \mathrm{sec}\right)\end{array}$} & $\square_{e}$ \\
\hline & & & & & & & \\
\hline core & 2.3 & 2.5 & 0.09 & 0.17 & 0.31 & 0.63 & 0.4 \\
\hline ITB & -0.9 & -0.9 & 0.03 & 0.01 & -0.12 & 0.09 & 0.03 \\
\hline outside & -116 & -149 & 17 & 3.2 & -203 & 49 & 11 \\
\hline \multicolumn{8}{|l|}{ TRANSP } \\
\hline core & -0.7 & -0.8 & 0.08 & 0.1 & 0.05 & 0.49 & 0.25 \\
\hline ITB & -1.8 & -2.3 & 0.36 & 1.1 & -0.2 & 0.39 & 1.1 \\
\hline outside & -3.1 & -4.3 & 0.49 & 1.8 & -6.0 & 0.47 & 1.7 \\
\hline
\end{tabular}




\section{Figure Captions}

Fig 1: C-Mod ITB discharge with off-axis ICRF heating throughout the discharge and central ICRF heating applied after ITB established. Summary traces are ICRF power, stored plasma energy, average density, neutron rate, ion temperature and toroidal rotation. Figure reproduced from Ref. 10, used with permission of the author.

Fig 2: Electron density $\left(10^{14} / \mathrm{cm}^{3}\right)$ versus radius $(r / a)$ showing evolution from ohmic and Lmode phases to RF H-mode and the ITB, density peaked phase. Timeslices show density becoming more peaked every $0.2 \mathrm{sec}$, from 0.5 to $1.2 \mathrm{sec}$, and then the central density showing a small decrease at $1.4 \mathrm{sec}$. The time of interest is $0.9 \mathrm{sec}$, before the ITB is established. The three radial locations for the gyrokinetic calculations are shown at $r / a \sim 0.25,0.45$ and 0.65 .

Figure 3. Comparison of the experimental (squares) and simulation (dimonds) time-to-peak data show good agreement for the assumed $\square^{p}$ profile (solid line). This indicates that the region of improved confinement is restricted to a narrow radial layer. Outside this region there is little evidence of improved transport. Figure reproduced from Ref. 10, used with permission of the author.

Figure 4. Real frequencies $\left(\sim 10^{6} / \mathrm{sec}\right.$, see frequency normalizations in Table II) of drift mode microturbulence from linear calculations for $k_{\square} \square_{i}$ from 0.1 to 80 . Calculations were fully electromagnetic, included four species and the complete nonadiabatic electron response for radial zones inside, at and outside the ITB region.

Figure 5. Growth rates $\left(\sim 10^{6} / \mathrm{sec}\right.$, see frequency normalizations in Table II) from linear calculations for $k_{\square} \square_{i}$ from 0.1 to 80 , the ITG-TEM, TEM and ETG range of wave vectors. ITGTEM is unstable outside the core, as is ETG. All modes are stable in the barrier region. The plasma core is found to have no well resolved, strongly unstable plasma modes. 
Figure 6. Normalized driving forces for drift mode microturbulence are balanced to stabilize instabilities inside and at the ITB, compared to outside the ITB. Calculations were fully electromagnetic and included nonadiabatic electron response. Greatest destabilizing ITG-TEM plasma response occurs with increases in $a_{r e f} \square T_{e} / T_{e}$.

Figure 7. a) In the barrier region ITG-TEM is linearly destabilized as $a_{r e f} \square n_{e} / n_{e}$ is decreased. The experimental error bars are $\sim 10 \%$, with the experimental value of $a_{r e f} \square n_{e} / n_{e}$ being 0.60 , far above the critical gradient destabilization point. For units, see frequency normalizations in Table II.

b) In the barrier region ITG-TEM is linearly destabilized as $a_{r e f} \square T_{e} / T_{e}$ is increased. The experimental error bars are $\sim 10 \%$, with the experimental value of $a_{r e f} \square T_{e} / T_{e}$ being 2.0 , very close to marginal stability. For units, see frequency normalizations in Table II.

Figure 8. Comparison of microturbulence levels is shown by displaying the volume-integrated magnitude of the square of the fluctuation potential from nonlinear calculations in the three plasma regions at the trigger time. Nonlinear electrostatic simulations of C-Mod before the ITB show the linear phase, followed by saturation. At the ITB region, the square of the fluctuation potential is reduced by two orders of magnitude, and by one order of magnitude in the plasma core, compared to the ITG-TEM unstable region outside the plasma core. A GAM (a device size and temperature dependent numerical mode) develops in the plasma core. The linear conclusion, that microturbulence is quiescent in the barrier region at this time, is also found in the nonlinear simulations.

Figure 9. Nonlinear results in the plasma core

a) Quasineutrality maintained in the plasma core to less than $1 \%$. 
b) The particle flux is outward, with electron and deuterium fluxes of similar magnitude and boron flux negligible. c) The heat flux is outward, with the deuterium heat flux being greater than the electron heat flux. The boron carries negligible heat flux.

Figure 10. Nonlinear results in the transport barrier region

a) Quasineutrality maintained in the plasma ITB region to much less than $1 \%$.

b) The particle flux from electrons and deuterium is inward, with electron and deuterium fluxes

of similar magnitude and boron flux being very small. c) The heat flux is outward, with the deuterium heat flux being about twice the electron heat flux. The boron carries negligible heat flux.

Figure 11. Nonlinear results outside the ITB region

a) Quasineutrality maintained outside the ITB region to $\sim 1 \%$.

b) The particle flux is inward, with electron flux $\sim 30 \%$ greater than the deuterium flux and boron flux negligible. c) The heat flux is outward, with the deuterium heat flux being about five times the electron heat flux. The boron carries negligible heat flux. 


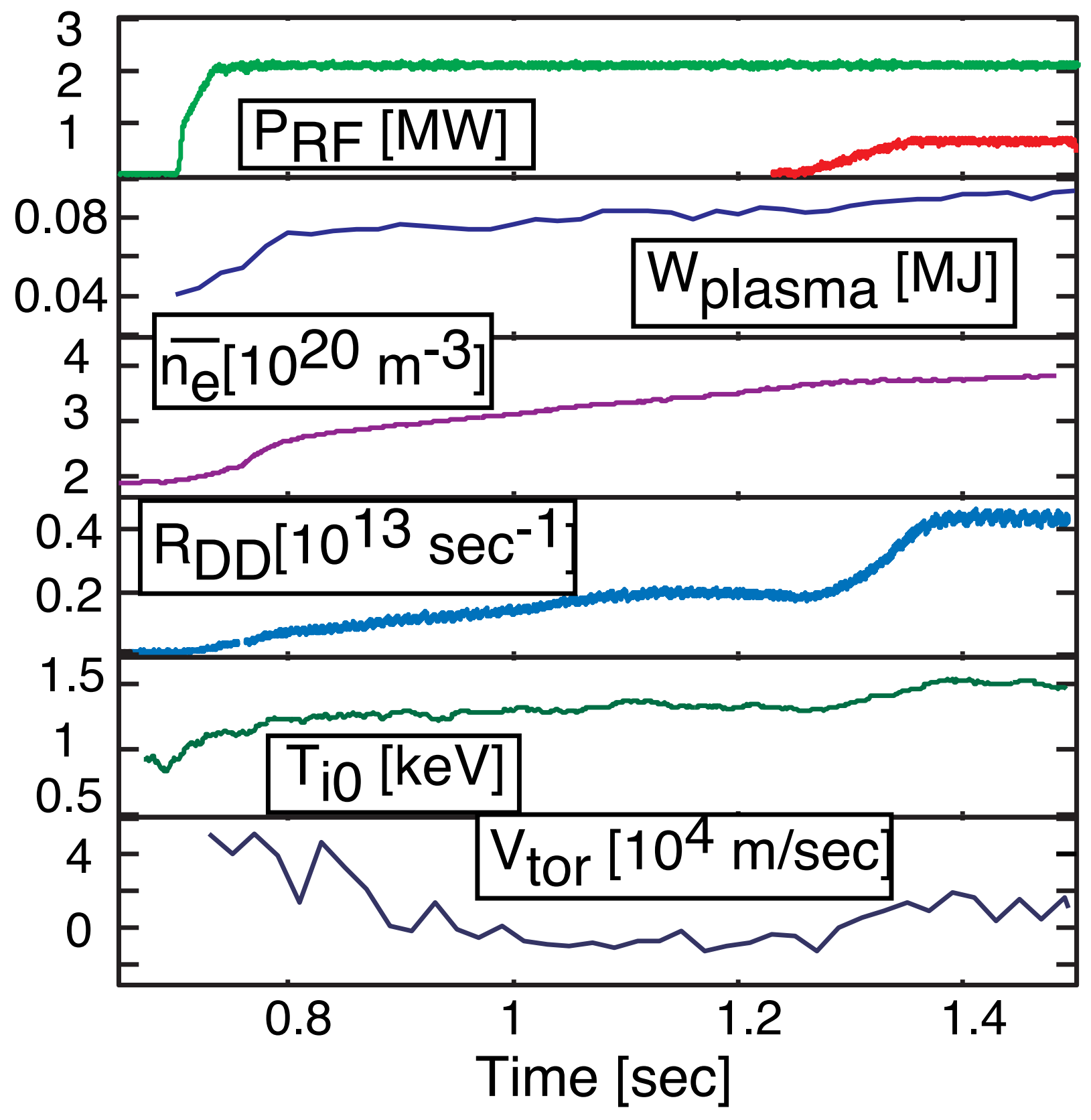

Fig 1 


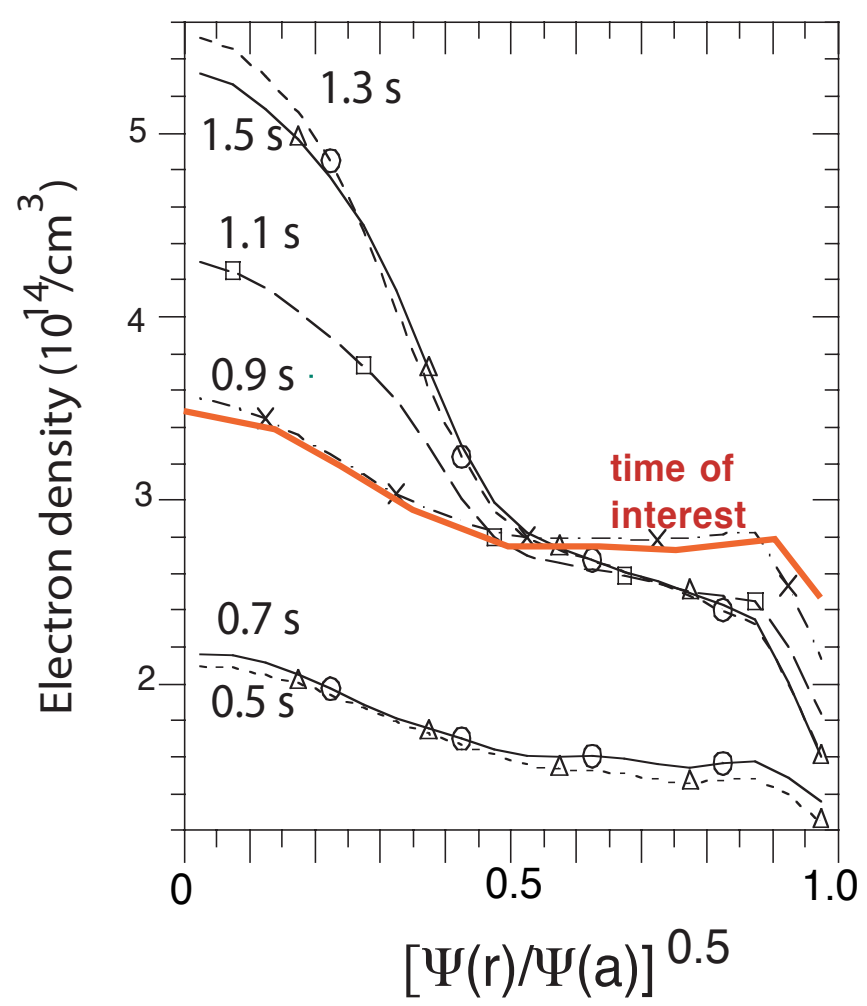

Fig. 2 


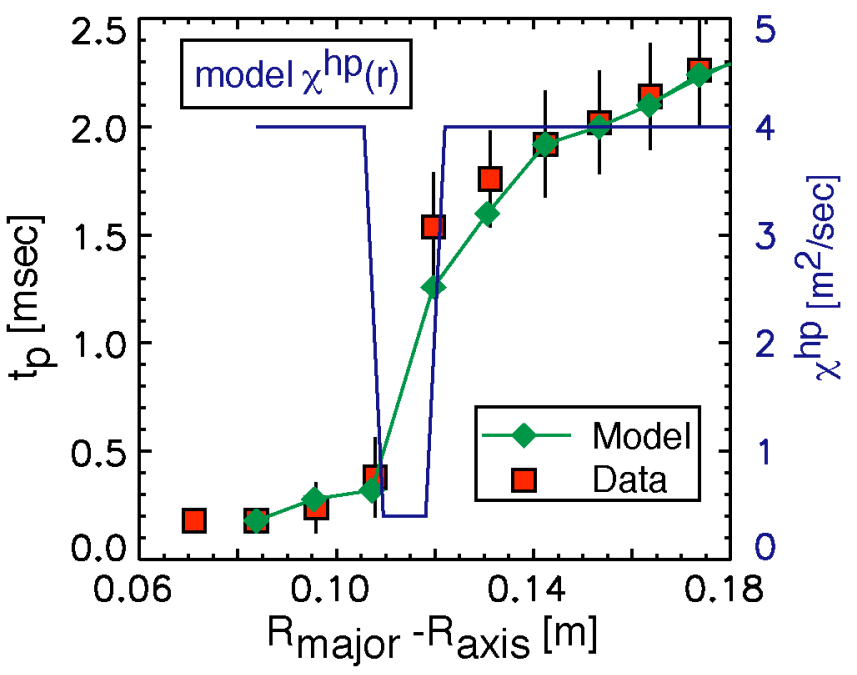

Fig 3 


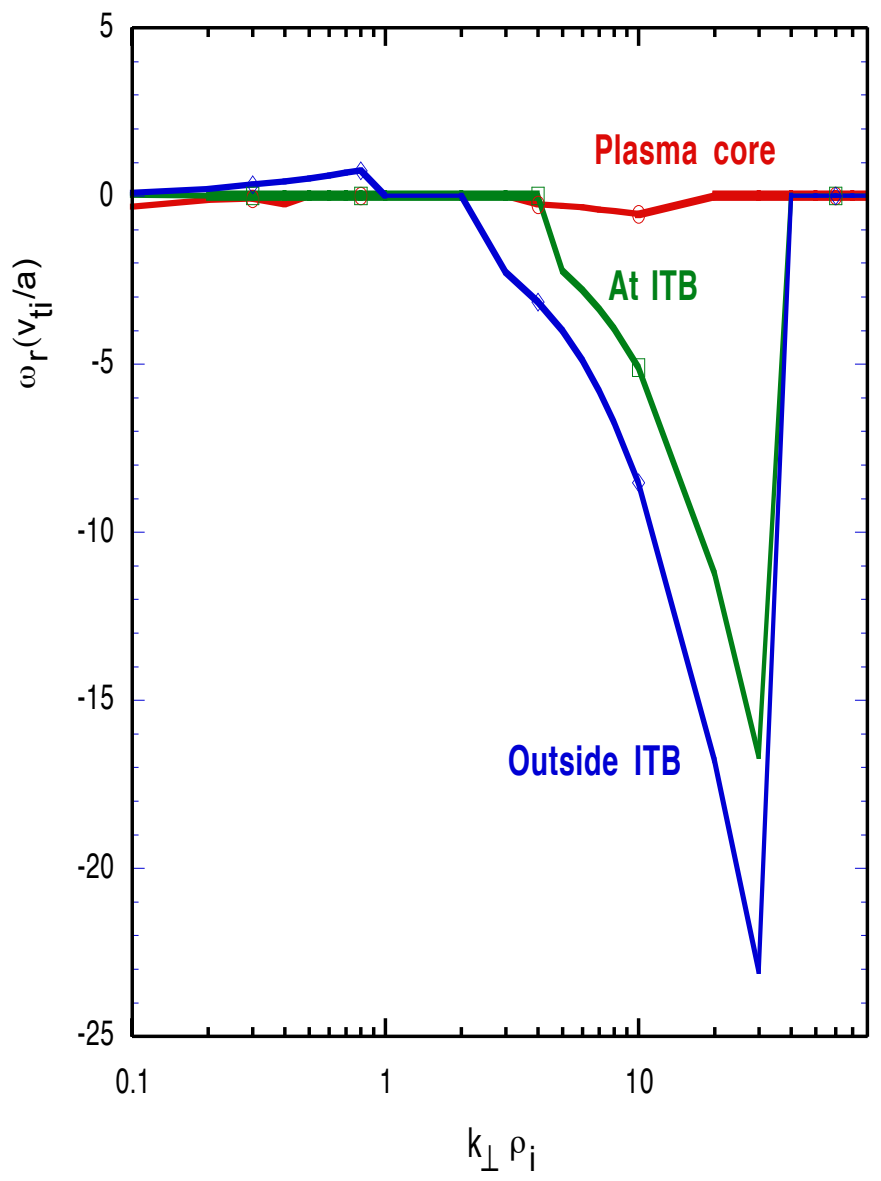

Fig 4 


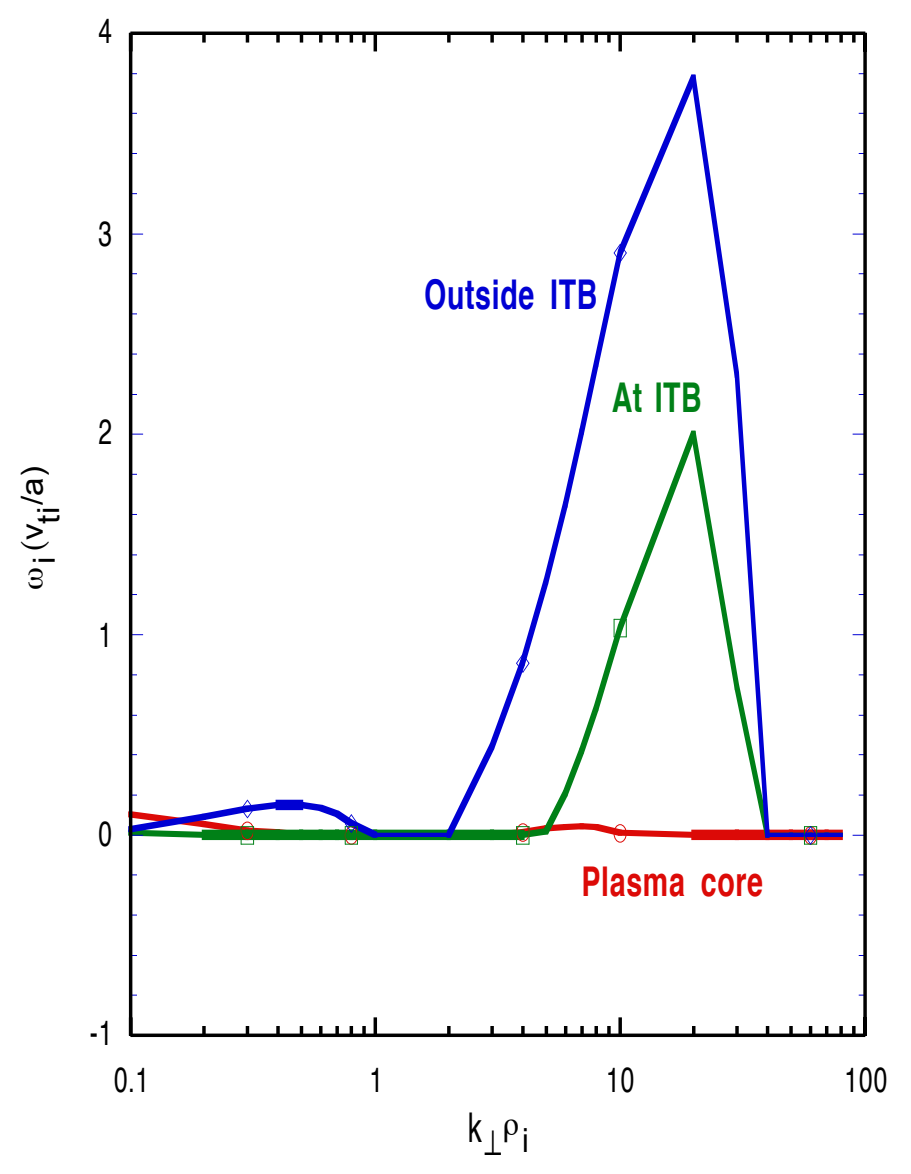

Fig 5 


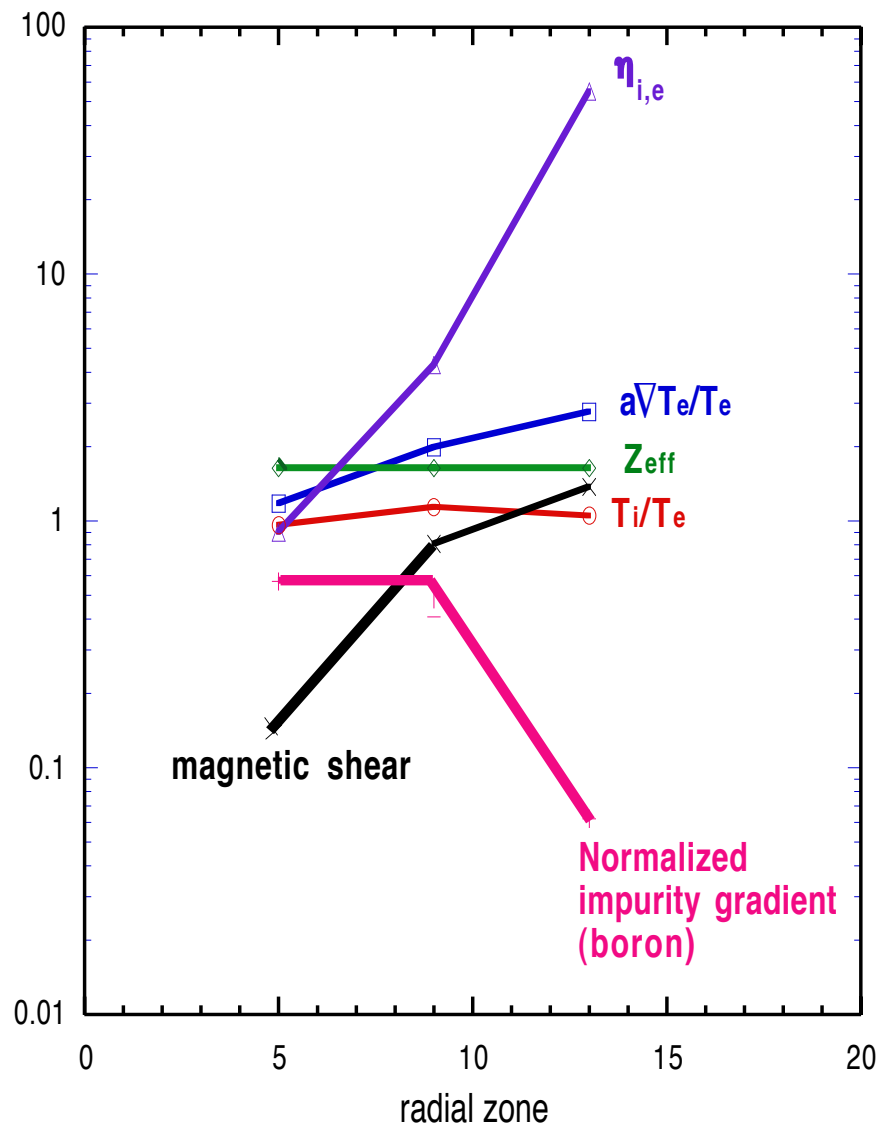

Fig 6 

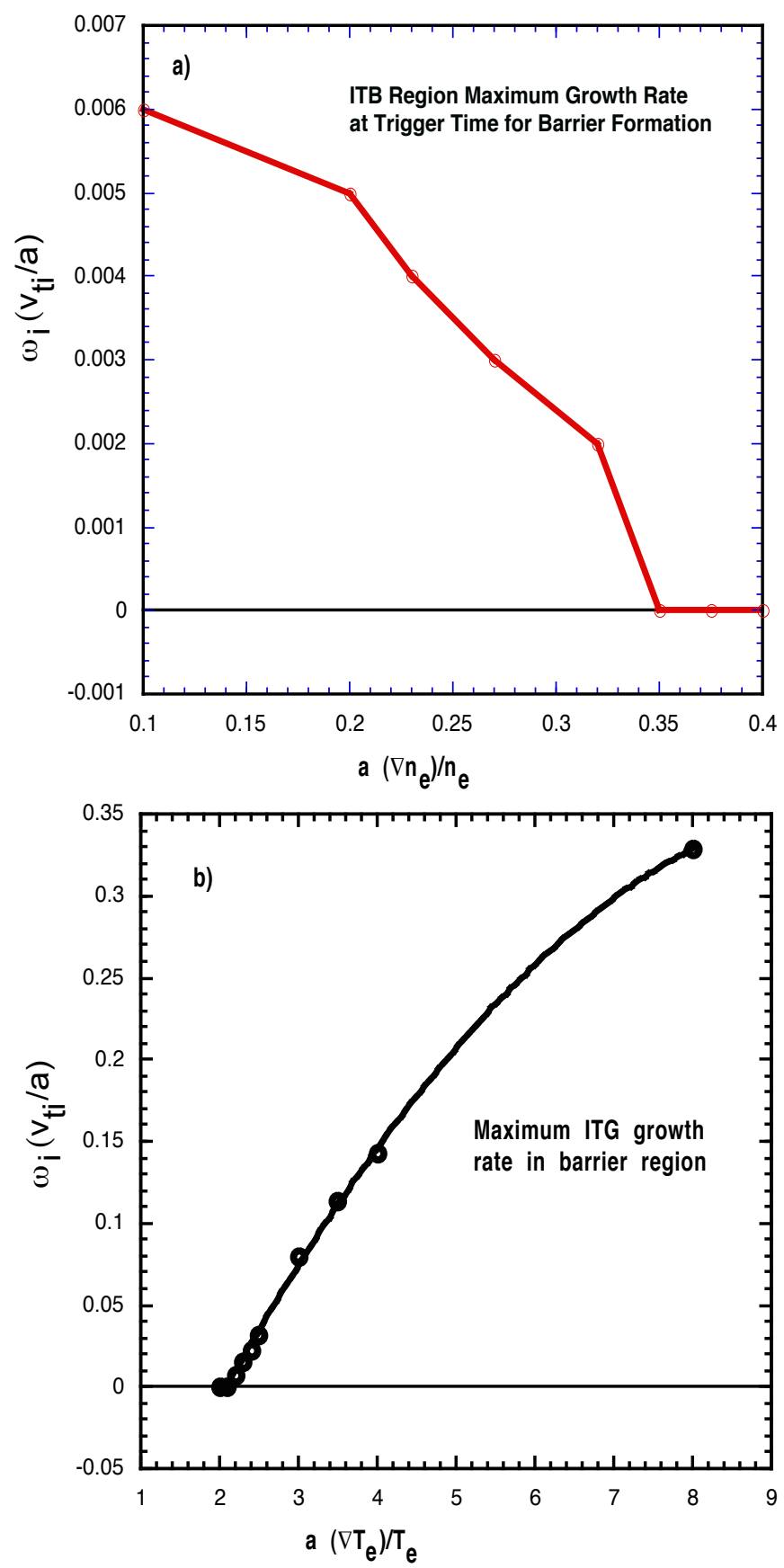

Fig 7 


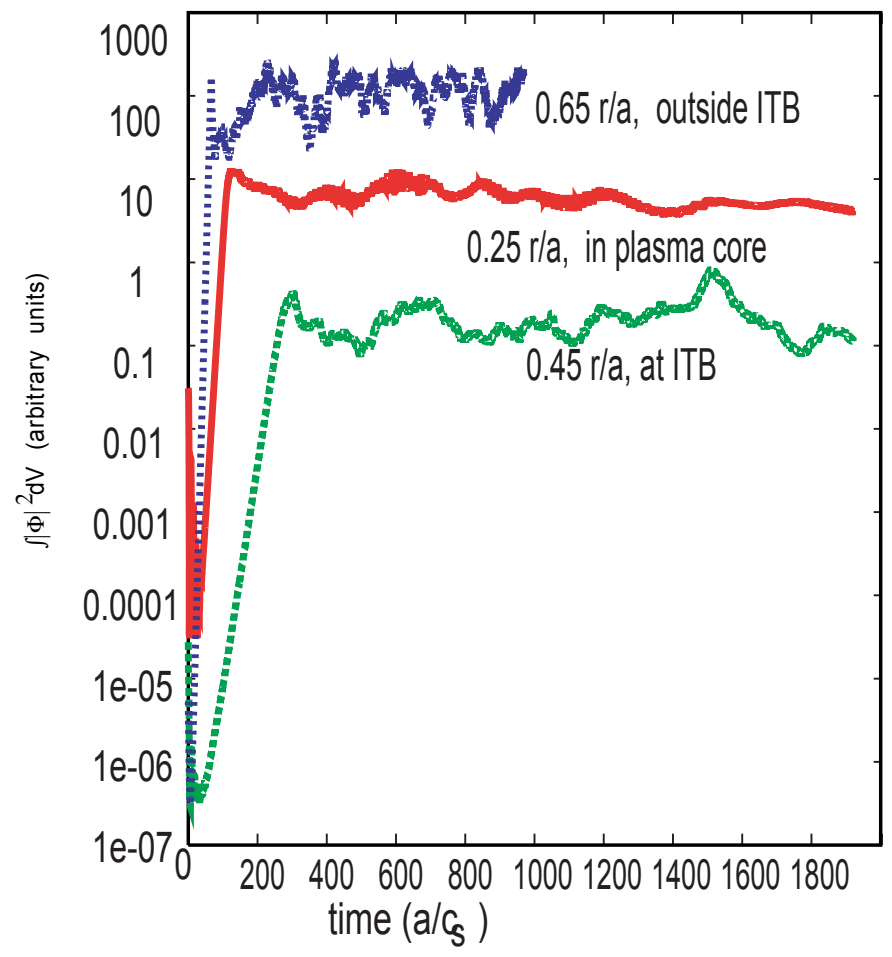

Fig. 8 

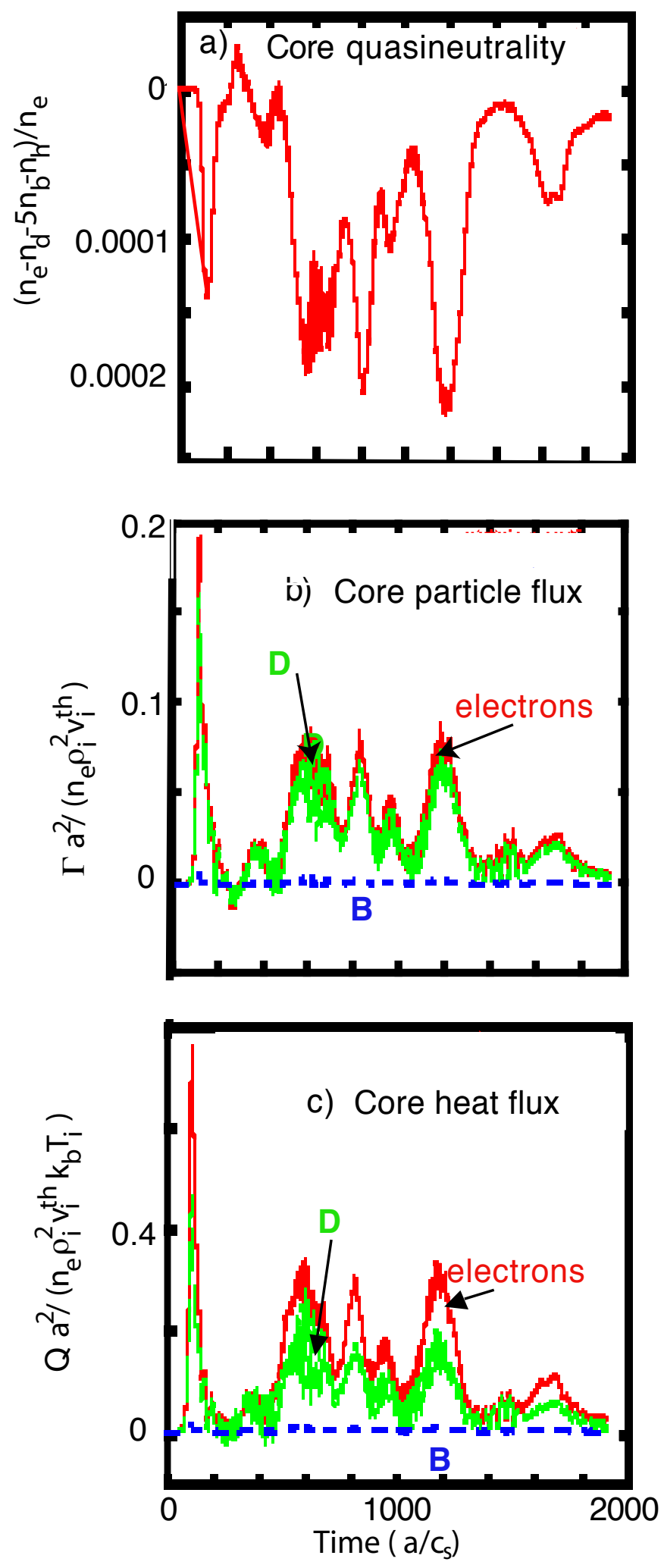

Fig 9 

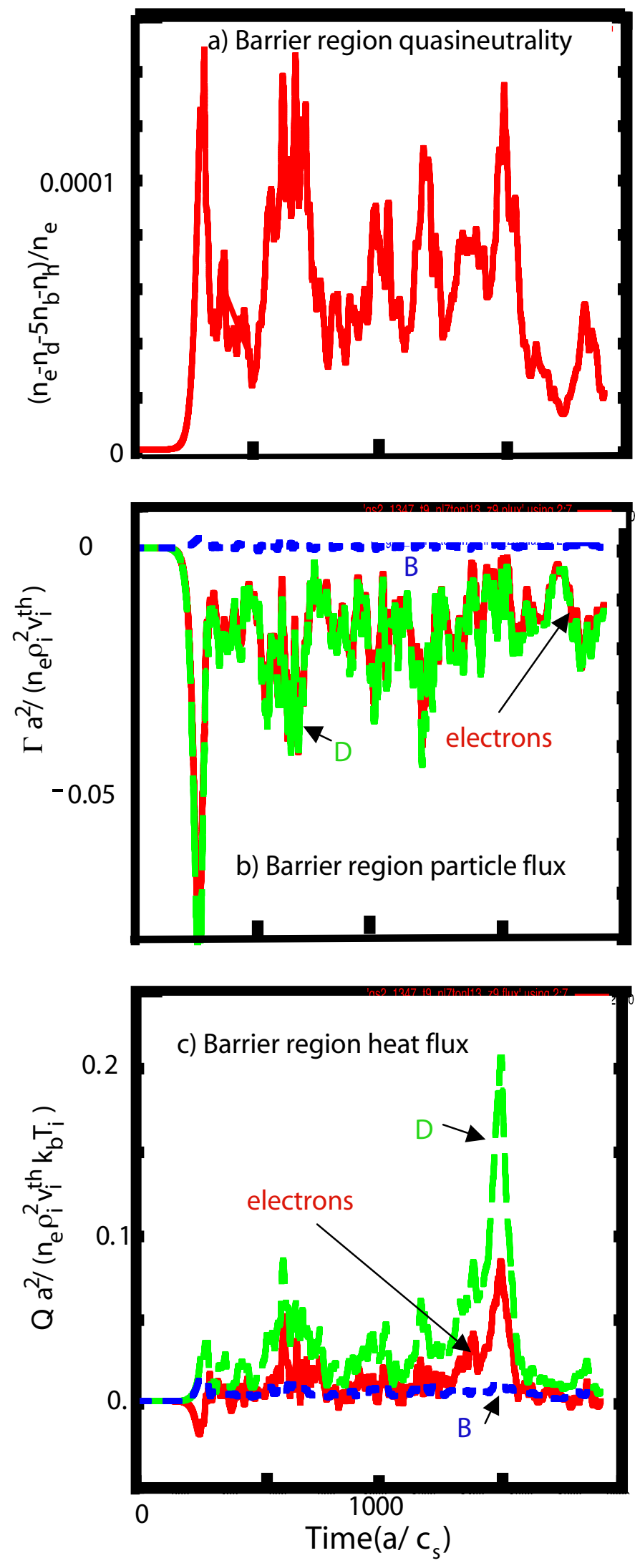

Fig 10 

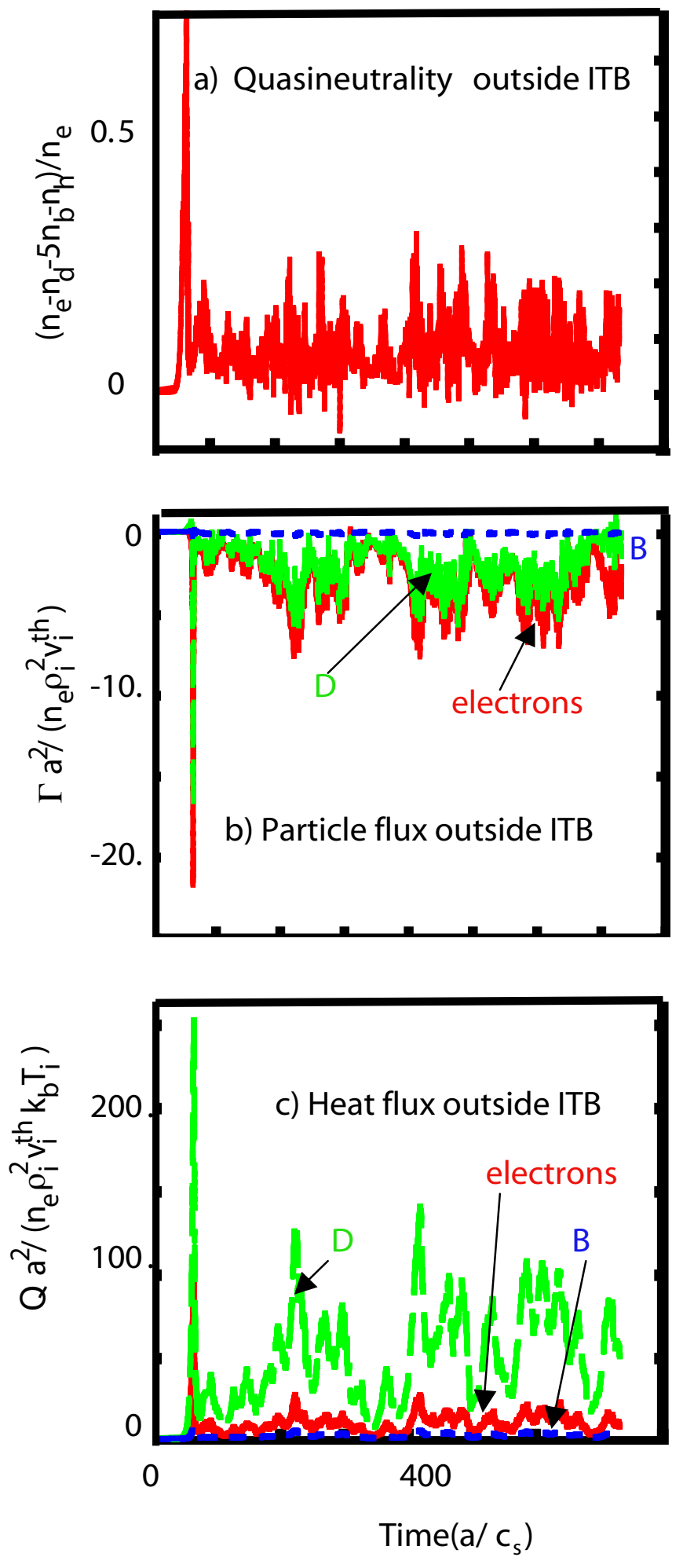

Fig11 


\section{External Distribution}

Plasma Research Laboratory, Australian National University, Australia

Professor I.R. Jones, Flinders University, Australia

Professor João Canalle, Instituto de Fisica DEQ/IF - UERJ, Brazil

Mr. Gerson O. Ludwig, Instituto Nacional de Pesquisas, Brazil

Dr. P.H. Sakanaka, Instituto Fisica, Brazil

The Librarian, Culham Laboratory, England

Mrs. S.A. Hutchinson, JET Library, England

Professor M.N. Bussac, Ecole Polytechnique, France

Librarian, Max-Planck-Institut für Plasmaphysik, Germany

Jolan Moldvai, Reports Library, Hungarian Academy of Sciences, Central Research Institute for Physics, Hungary

Dr. P. Kaw, Institute for Plasma Research, India

Ms. P.J. Pathak, Librarian, Institute for Plasma Research, India

Ms. Clelia De Palo, Associazione EURATOM-ENEA, Italy

Dr. G. Grosso, Instituto di Fisica del Plasma, Italy

Librarian, Naka Fusion Research Establishment, JAERI, Japan

Library, Laboratory for Complex Energy Processes, Institute for Advanced Study, Kyoto University, Japan

Research Information Center, National Institute for Fusion Science, Japan

Dr. O. Mitarai, Kyushu Tokai University, Japan

Dr. Jiangang Li, Institute of Plasma Physics, Chinese Academy of Sciences, People's Republic of China

Professor Yuping Huo, School of Physical Science and Technology, People's Republic of China

Library, Academia Sinica, Institute of Plasma Physics, People's Republic of China

Librarian, Institute of Physics, Chinese Academy of Sciences, People's Republic of China

Dr. S. Mirnov, TRINITI, Troitsk, Russian Federation, Russia

Dr. V.S. Strelkov, Kurchatov Institute, Russian Federation, Russia

Professor Peter Lukac, Katedra Fyziky Plazmy MFF UK, Mlynska dolina F-2, Komenskeho Univerzita, SK-842 15 Bratislava, Slovakia

Dr. G.S. Lee, Korea Basic Science Institute, South Korea

Institute for Plasma Research, University of Maryland, USA

Librarian, Fusion Energy Division, Oak Ridge National Laboratory, USA

Librarian, Institute of Fusion Studies, University of Texas, USA

Librarian, Magnetic Fusion Program, Lawrence Livermore National Laboratory, USA

Library, General Atomics, USA

Plasma Physics Group, Fusion Energy Research Program, University of California at San Diego, USA

Plasma Physics Library, Columbia University, USA

Alkesh Punjabi, Center for Fusion Research and Training, Hampton University, USA

Dr. W.M. Stacey, Fusion Research Center, Georgia Institute of Technology, USA

Dr. John Willis, U.S. Department of Energy, Office of Fusion Energy Sciences, USA

Mr. Paul H. Wright, Indianapolis, Indiana, USA 
The Princeton Plasma Physics Laboratory is operated by Princeton University under contract with the U.S. Department of Energy.

\author{
Information Services \\ Princeton Plasma Physics Laboratory \\ P.O. Box 451 \\ Princeton, NJ 08543
}

Phone: 609-243-2750

Fax: 609-243-2751

e-mail: pppl_info@pppl.gov

Internet Address: http://www.pppl.gov 